\title{
Grazing-incidence antireflection films. II. Alternate techniques and general multilayer theory
}

\author{
J. P. Hannon, N. V. Hung, and G. T. Trammell \\ Physics Department, Rice University, Houston, Texas 77251 \\ E. Gerdau, M. Mueller, R. Rüffer, and H. Winkler \\ Institut für Experimentalphysik, Universität Hamburg, Hamburg, Germany
}

(Received 9 May 1984)

\begin{abstract}
In this second of two papers on nonresonant grazing-incidence antireflection films, we examine alternate techniques for achieving high-efficiency antireflection films for suppressing hard-x-ray reflections: half-wave films, layered ultrathin films, general multilayer techniques, and tapered impedance films.
\end{abstract}

\section{INTRODUCTION}

In the preceding paper $I,{ }^{1}$ we discussed the possibility of grazing-incidence antireflection films for creating high-efficiency antireflection coatings for near-grazingincidence reflection of hard $\mathrm{x}$ rays and $\gamma$ rays. Our motivation is the possible application of producing "ultranarrow" bandpass filters for synchrotron radiation with frequency widths $\approx 10^{-8}-10^{-6} \mathrm{eV}$, giving a unique high-resolution, long-coherence-length $\mathrm{x}$-ray source for probing inelastic excitations and opening new possibilities in X-ray interferometry. In paper I we developed the general theory and examined the simplest ideas for achieving antireflection films-impedance-matched quarter-wave films and damping-stabilized solutions-which can both be obtained by coating a high-density mirror with a single lower-density film.

In this paper we discuss a number of alternate techniques for strongly suppressing $x$-ray reflections: halfwave films, layered ultrathin films, general multilayer techniques, and tapered impedance films. Sections II and III are direct applications of the development given in paper $I^{1}{ }^{1}$ and Sec. IV gives a general multilayer theory for grazing-incidence antireflection (GIAR) films.

\section{II. $\lambda / 2$ FILMS}

If the electron density of the film is higher than that of the backing so that now $\phi_{c 1}>\phi_{c 2}$, then a true antireflection coating is impossible: Due to the backing, the electron-density change at the $0-1$ interface is necessarily greater than at the 1-2 interface, and the impedancematch condition $r_{01}=r_{12} \exp \left(-2 g_{1}^{\prime \prime} l_{1}\right)$ can no longer be satisfied. Nevertheless, destructive interference still occurs, now at a half-wave condition. Because of the lack of impedance match, the reductions are rather modest but, for some purposes, these may be sufficient.

As a typical example, we consider an Fe film on Si, reflecting $14.4-\mathrm{keV}$ radiation $\left(\phi_{c 1}=3.8 \mathrm{mrad}, \phi_{c 2}=2.1\right.$ mrad). The reflection amplitude is given by the general expression, Eq. (9) or (17) of paper I. Figure 1 gives the rocking curves for the interface reflection amplitudes $r_{01}$ and $r_{12} \exp \left(-2 g_{1}^{\prime \prime} l_{1}\right)$ and for the corresponding phases $\phi_{01}$ and $\phi_{12}$.

The behavior of the reflection amplitudes has important modifications from the low-density-film case (Fig. 6 of paper I) because now $\phi_{c 1}>\phi_{c 2}$ : As before, $r_{01}$ has neartotal reflection for $\phi<\phi_{c 1}$, while $r_{12}$ has a near-total reflection region for $\phi_{c 2} \leq \phi \leq \phi_{c 1}$. However, when damping effects are included, the lower interface reflection $r_{12} \exp \left(-2 g_{1}^{\prime \prime} l_{1}\right)$ peaks only near $\phi_{c 1}$ because there is strong primary extinction for $\phi<\phi_{c 1}$, the total reflection region for the upper medium. Because $\phi_{c 2}<\phi_{c 1}$, $r_{12} \exp \left(-2 g_{1}^{\prime \prime} l_{1}\right)$ is everywhere less than $r_{01}$ and there can be no impedance match. For interference, the region of interest is $\phi>\phi_{c 1}$, where deep penetration into film 1 occurs, and in this region $r_{01}$ and $r_{12} \exp \left(-2 g_{1}^{\prime \prime} l_{1}\right)$ slope together, with the difference remaining fairly small, $\approx \phi_{c 2}^{2} / 4 \phi^{2}$.

The rocking curves for the phases $\phi_{01}$ and $\phi_{12}$ of the interface reflections are shown in Fig. 1(b). As for the lowdensity case, $\phi_{01}$ increases rapidly from $-\pi \rightarrow 0$ as $\phi \propto 0 \rightarrow \phi_{c 1}$, but now, $\phi_{12}$ increases from $0 \rightarrow \pi$ as $\phi \propto \phi_{c 2} \rightarrow \phi_{c 1}$. The phases are constant outside of these regions, and in the interference region $\phi>\phi_{c 1}$, the phase difference is $\phi_{01}-\phi_{12} \simeq-\pi$, corresponding to the expected opposite signs of $R_{01}$ and $R_{12}$.

The condition for destructive interference is again given by Eq. (21) of paper I, but now, $\phi_{01}-\phi_{12} \simeq-\pi$ and the required film thickness is a "half-wave film,"

$$
l_{1}^{(2 n+1) \pi}(\phi) \simeq n \lambda / 2\left(\phi^{2}-\phi_{c 1}^{2}\right)^{1 / 2} .
$$

A simple estimate can be given for the strength of the impedance mismatch which occurs at the interference minima: Conceptually taking a small gap $\Delta l \rightarrow 0$ between film 1 and medium 2, then, in the single scattering approximation, the total reflection will be a superposition of three vacuum-interface reflections, giving

$$
R_{\min } \simeq r_{01}\left(1-e^{-2 g_{1}^{\prime \prime} l_{1}}\right)+r_{02} e^{-2 g_{1}^{\prime \prime} l_{1}} .
$$

The first contribution gives the reflection from a free film at the $\lambda / 2$ condition, and here the impedance mismatch is due entirely to photoabsorption. The second contribution 


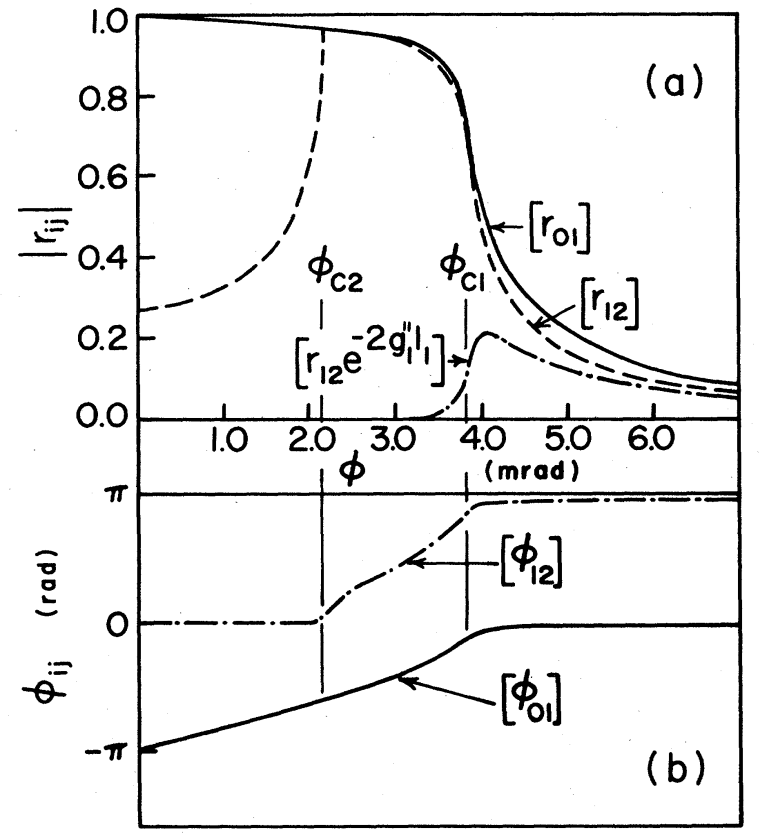

FIG. 1. (a) Interface reflection amplitudes, and (b) phases vs the incidence angle $\phi$ for $185-\AA \mathrm{Fe}$ film on $\mathrm{Si}$, reflecting 14.4$\mathrm{keV}$ radiation.

is the vacuum-substrate reflection, with a photoabsorption reduction for propagation through film 1. Both contributions are positive and add constructively to increase the total reflection.

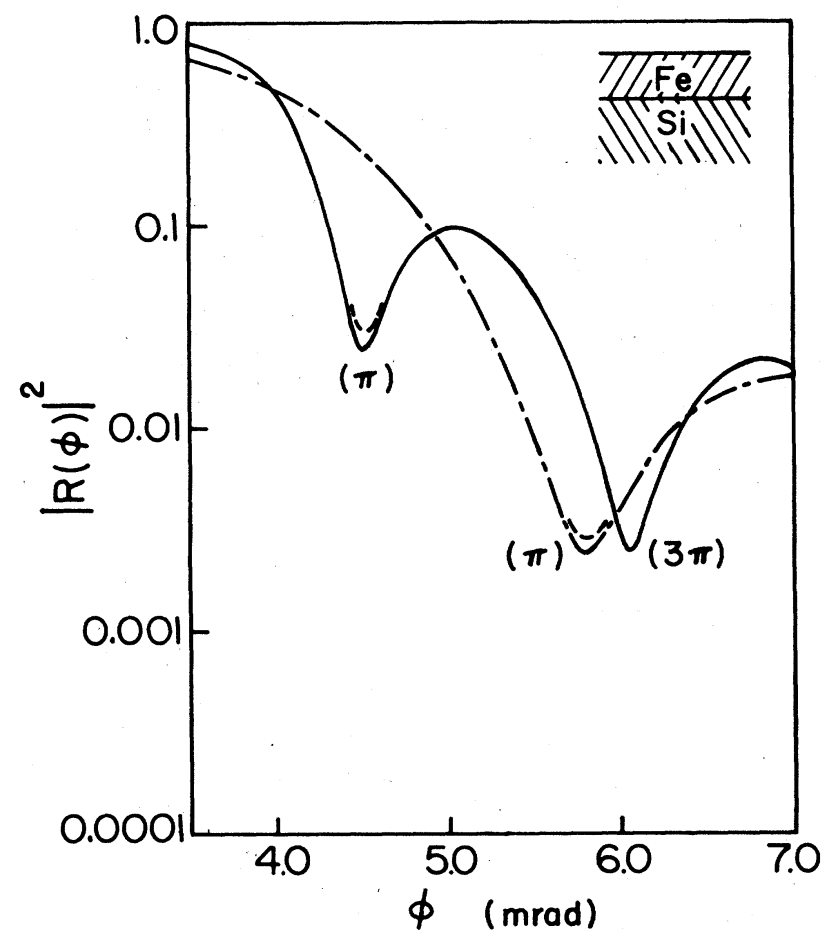

FIG. 2. Rocking curves $|R|^{2}$ vs $\phi$ for $185-\AA$ Fe on Si (solid curve) and $100-\AA \mathrm{Fe}$ on $\mathrm{Si}$ (dashed curve), reflecting $14.4-\mathrm{keV}$ radiation.
From this estimate it is clear that the reflection can be minimized by taking the lowest-density substrate possible to reduce $r_{02}$ and by taking a thinner film $l_{1}$ and a larger incidence angle $\phi$ to reduce the path length in film 1 and the photoabsorption factor $\left[1-\exp \left(-2 g_{1}^{\prime \prime} l_{1}\right)\right]$. The increased $\phi$ also acts to further reduce $r_{02}$.

As an example, for a $185-\AA$ Fe film on $\mathrm{Si}$ and $14.4-\mathrm{keV}$ radiation, the first $\lambda / 2$ minimum occurs for $\phi_{0}=4.5 \mathrm{mrad}$ and Eq. (2) gives a reflectivity $|R|^{2} \simeq 0.02$ in a region where normally $|R|^{2} \simeq 0.10$. On the other hand, for a 100 - $\AA$ film, $\phi_{0}=5.7 \mathrm{mrad}$ and $|R|^{2} \simeq 2 \times 10^{-3}$ in a region where normally $|R|_{\mathrm{Fe}}^{2} \simeq 0.01$. In both cases the reduction factor $|R|^{2} /|R|_{\mathrm{Fe}}^{2} \approx \frac{1}{5}$. These estimates are in good agreement with the minima of the exact rocking curves shown in Fig. 2.

There is a practical advantage to $\lambda / 2$ films: In contrast to $\lambda / 4-$ GIAR films, no careful matching of densities and thicknesses is required since any thickness high-density film on a low-density backing will have a series of interference minima for $\phi>\phi_{c 1}$. Although the reduction factors are not very pronounced, for some applications they may be sufficient.

\section{ULTRATHIN FILMS}

Our primary interest in GIAR films is for resonant $\gamma$-ray optics. For the ${ }^{57} \mathrm{Fe} 14.4-\mathrm{keV}$ transition, the resonance scattering is an order of magnitude greater than the nonresonance electronic scattering. Because of this difference in scattering strengths, another technique for reducing the electronic scattering is to use an "ultrathin" film $\approx 10-20 \AA$, which is "thin" for the electronic scattering but still "thick" for the resonant nuclear scattering.

\section{A. Single ultrathin film on low-density backing}

For a single ultrathin film on a low-density backing, the reflected wave amplitude is still given by Eq. (9) or (17) of paper I. On a low-density backing, $R_{12} \simeq-R_{01}$ and there is a strong reduction in scattering if the film is sufficiently thin, so that the phase factor $\exp \left(i 2 g_{1} l_{1}\right) \simeq 1$.

The condition for an ultrathin (UT) film is then

$$
\begin{aligned}
l_{1<l_{\mathrm{UT}}(\phi)} & =\left(2\left|g_{1}\right|\right)^{-1} \\
& =\hbar /\left\{2\left[\left(\phi^{2}-\phi_{c 1}^{2}\right)^{2}+\left(\hbar / l_{A}\right)^{2}\right]^{1 / 4}\right\} .
\end{aligned}
$$

The most favorable case is for $\phi \approx \phi_{c 1}$, where the refracted wave is nearly parallel to the surface. Figure 3 gives $l_{\mathrm{UT}}(\phi)$ for the ${ }^{57} \mathrm{Fe}$ case which has a peak value $\approx 81 \mathrm{~A}$ at $\phi=\phi_{c 1}$.

For films with $l_{1} \ll l_{\mathrm{UT}}(\phi)$, there is little extinction or phase change across the film. All atoms then reflect in phase into the reflection channel and the reflected amplitude increases with increasing thickness for thicknesses up to $\approx l_{\mathrm{UT}}$. Thus, for $l_{1} \ll l_{\mathrm{UT}}(\phi)$, the reflection amplitude is reduced from $r_{01}$ approximately by a factor $l_{1} / l_{\mathrm{UT}}(\phi)$.

More exactly, if $l_{1}<l_{\mathrm{UT}}(\phi)$, the reflection amplitude, Eq. (9) of paper I reduces to 


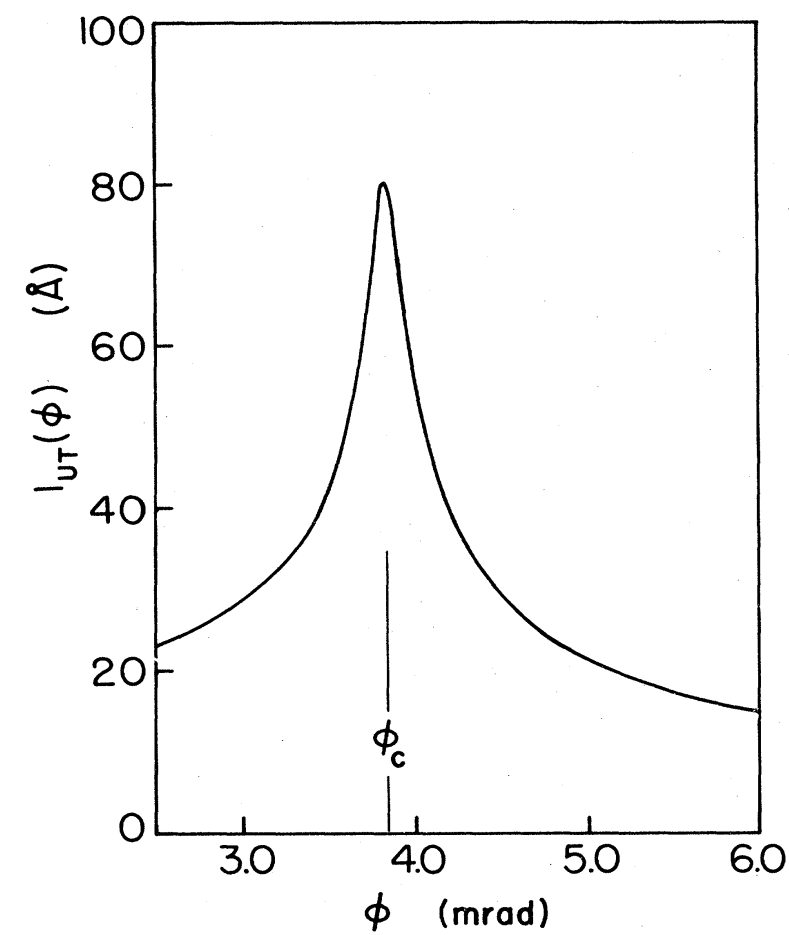

FIG. 3. Ultrathin-film limiting thickness $l_{\mathrm{UT}}(\phi)$ vs $\phi$ for $\mathrm{Fe}$, reflecting $14.4-\mathrm{keV}$ radiation.

$$
R(\phi) \simeq i F\left(l_{1}\right)+R_{02},
$$

where

$$
F\left(l_{1}\right)=n \lambda l_{1} f_{e} / \phi=-\left(\pi \phi_{c}^{2} l_{1} / \lambda \phi\right)\left(1-i \lambda / \phi_{c}^{2} l_{A}\right) .
$$

The first contribution to $R, i F\left(l_{1}\right)$, gives the scattering from a free UT film, while the second contribution $R_{02}$ gives the scattering from the uncoated backing.

Now, $i F\left(l_{1}\right)$ is just the usual Born approximation for scattering from a "single plane" (see Appendix A of paper I). The scattering is necessarily $90^{\circ}$ out of phase with the primary wave [so that in the forward direction the imaginary contribution to $F\left(l_{1}\right)$, which is due to the total absorption cross section, will give a wave $180^{\circ}$ out of phase with the primary, leading to extinction]. Because of the $90^{\circ}$ phase shift and because $F\left(l_{1}\right)$ and $R_{02}$ are both essentially real (in the region $\phi>\phi_{c 2}$ ), there is little interference between the UT film and substrate reflections, and $|R|^{2} \simeq F\left(l_{1}\right)^{2}+R_{02}^{2}$. To minimize the backing contribution, the substrate should be taken with the lowest density possible. As a specific example, consider $10 \AA$ of $\mathrm{Fe}$ on a Be backing reflecting $14.4-\mathrm{keV}$ radiation. Then for $\phi \approx \phi_{c 1}, F\left(l_{1}\right)^{2} \simeq 0.02$ and $R_{02} \simeq 0.003$, giving a reflection $|R|^{2} \simeq 0.02$ in a region of near-total reflection from a pure Fe mirror.

Figure 4 gives the exact rocking curve $|R(\phi)|^{2}$ for a $10-\AA \mathrm{Fe}$ film on $\mathrm{Be}$, and also for $\left|R_{01}(\phi)\right|^{2}$ (pure $\mathrm{Fe}$ ) and $\left|R_{02}(\phi)\right|^{2}$ (pure Be). In the region $\phi \approx \phi_{c 1}$ we see that $|R|^{2}$ is reduced from $\left|R_{01}\right|^{2}$ by a factor $\simeq \frac{1}{30}$, which is a considerably stronger reduction factor than for a $\lambda / 2$ film.

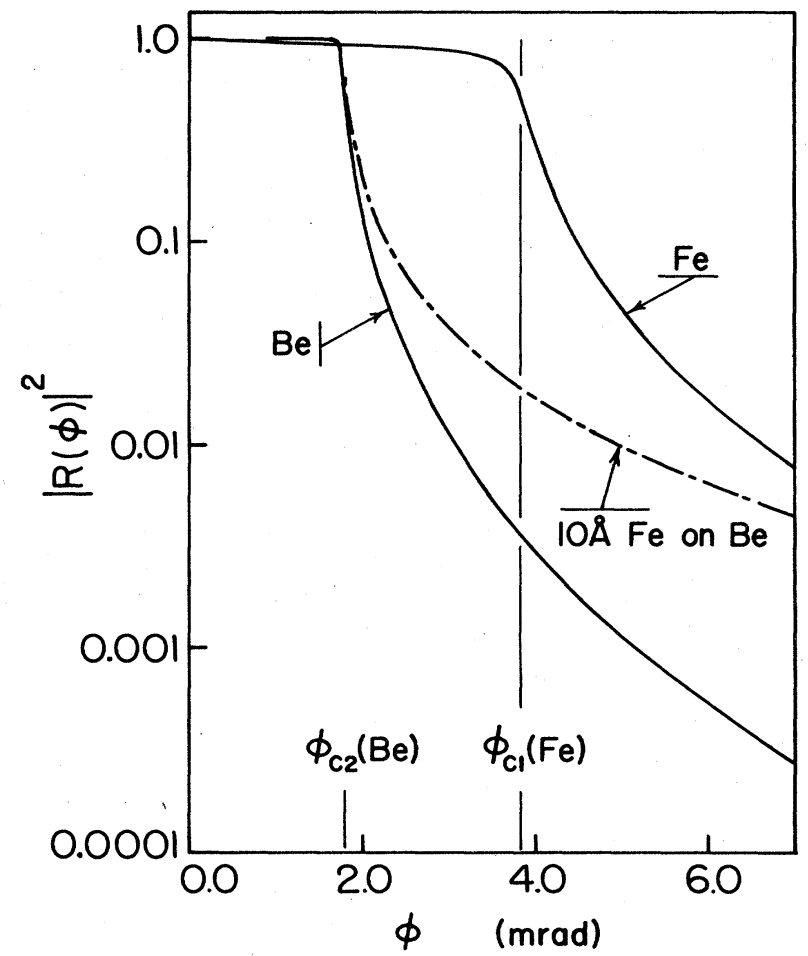

FIG. 4. Rocking curve $|R|^{2}$ vs $\phi$ for a single ultrathin film of $10-\AA \mathrm{Fe}$ on $\mathrm{Be}$, reflecting $14.4-\mathrm{keV}$ radiation (dashed curve). The solid curves give the reflectivities for pure $\mathrm{Fe}$ and pure $\mathrm{Be}$.

\section{B. UT-film interference techniques}

Although a fairly strong reduction can be achieved with a single ultrathin film, the reflectivity is still $|R|^{2} \geq 10^{-2}$, and for applications such as synchrotron filtering, we would like $|R|^{2} \lesssim 10^{-3}$. To achieve such a strong reduction it is necessary to use interference.

One simple method is to use two UT layers $l_{1}$ and $l_{3}$, separated by a low-density film $l_{2}$ and coated on a lowdensity backing, as indicated in Fig. 5(a). In the UT limit, the multilayer reflection amplitude is approximately

$$
R(\phi) \simeq i F\left(l_{1}\right)+R_{02}+i F\left(l_{3}\right) e^{i 2 g_{2} l_{2}}+R_{24} e^{i 2 g_{2} l_{2}} .
$$

Here, $i F\left(l_{1}\right)$ and $i F\left(l_{3}\right)$ are the scattering contributions from the two (free) UT films, $R_{02}$ is the reflection from a vacuum-medium-2 interface, and $R_{24}$ is the reflection from a medium-2-medium-4 interface. As noted before, the UT limit holds best for $\phi \simeq \phi_{c 1}\left(=\phi_{c 3}\right)$.

Because the UT reflections are $90^{\circ}$ out of phase with the 0-2,2-4 interface reflections, there is separate interference between the two UT reflections and between the two interface reflections. If medium 4 is taken at higher density than medium 2, then both $R_{24}$ and $R_{02}>0$, and there will be simultaneous destructive interference between the two UT reflections and between the two interface reflections when a quarter-wave condition holds for $l_{2}$ so that $\exp \left(i 2 g_{2} l_{2}\right)=-1$. In principle, we could take the density of medium 2, such that an impedance match would also òccur at this angle, giving $R_{02} \approx R_{24}$. However, it is un- 


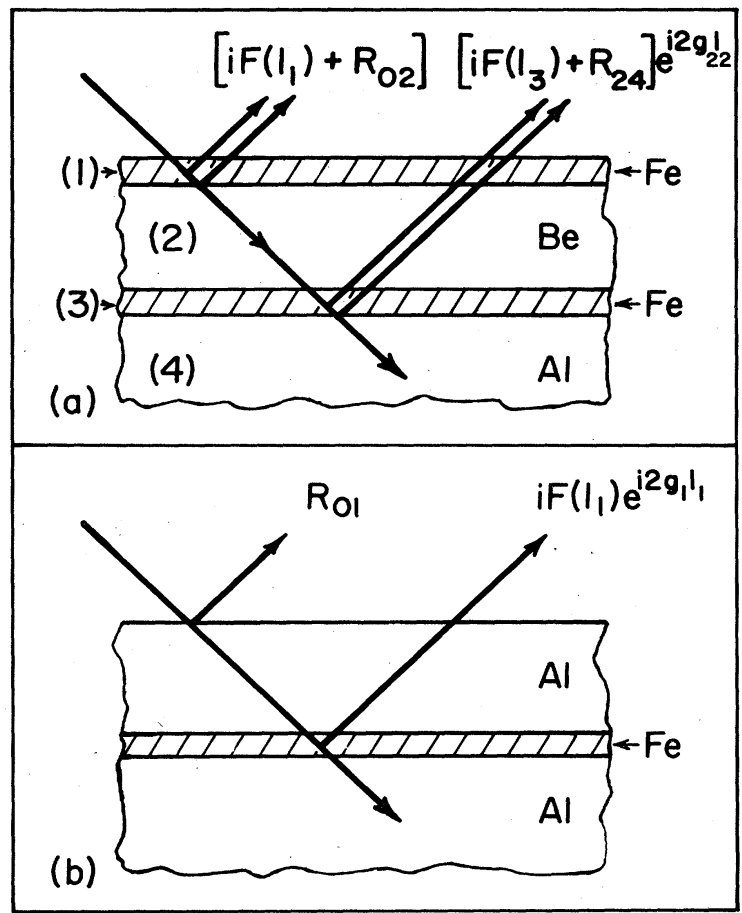

FIG. 5. Geometry for (a) two-layered ultrathin films, and (b) for a single ultrathin film coated with a $3 \lambda / 8$ film.

necessary to strive for exact cancellations here because all amplitudes are already small, and with only partial cancellations, it should still be easy to achieve $|R|^{2} \leq 10^{-3}$. Thus, in practice, it should suffice to take media $\widetilde{2}$ and 4 as any two low-density media, with critical angles $\phi_{c 22}<\phi_{c 4}<<\phi_{c 1}$ so that $R_{02}$ and $R_{24}$ are small for $\phi \approx \phi_{c 1}$ (where the UT limit holds best) and with $l_{2} \simeq \lambda / 4\left(\phi_{c 1}^{2}-\phi_{c 2}^{2}\right)^{1 / 2}$ so that a $\lambda / 4$ condition holds for $\phi \approx \phi_{c 1}$. Also, the UT film 2 needs to be somewhat thicker than the UT film 1 to offset the small extinction effects in films 1 and 2.

As an example, in Fig. 6 we give the exact rocking curve $|R(\phi)|^{2}$ for a $10-\AA \mathrm{Fe}-64-\AA \mathrm{Be}-12-\AA \mathrm{Fe}-\mathrm{Al}$ multilayer which has a very pronounced minimum $|R|^{2}<10^{-4}$ for $\phi \simeq 3.7 \mathrm{mrad}$. The dashed curve in Fig. 18 gives the corresponding results for a $15-\AA \mathrm{Fe}-64$ $\AA \mathrm{Be}-17-\AA \mathrm{Fe}-\mathrm{Al}$ multilayer which also has a pronounced minimum, but shifted to $\phi \simeq 3.6 \mathrm{mrad}$ by the thicker films. An exact solution procedure to determine the optimum $l_{1}, l_{2}$, and $l_{3}$ is given in the following section. Such UT-GIAR films should be capable of giving as strong a suppression of $\mathrm{x}$-ray reflection as $\lambda / 4$-GIAR films, and in practice it may be easier to achieve strong suppression with UT-GIAR films because it is unnecessary to try to impedance match at the $\lambda / 4$ condition.

An alternate UT-film interference technique is a $3 \lambda / 8$ coated UT film indicated in Fig. 5(b): An UT film of Fe is coated on a low-density mirror, say, $\mathrm{Al}$ and then a film of the mirror material (Al) is coated on top. In the UTfilm limit, the reflection amplitude is then approximately

$$
R \simeq R_{01}+e^{i 2 g_{1} l_{1}} i F\left(l_{2}\right) .
$$

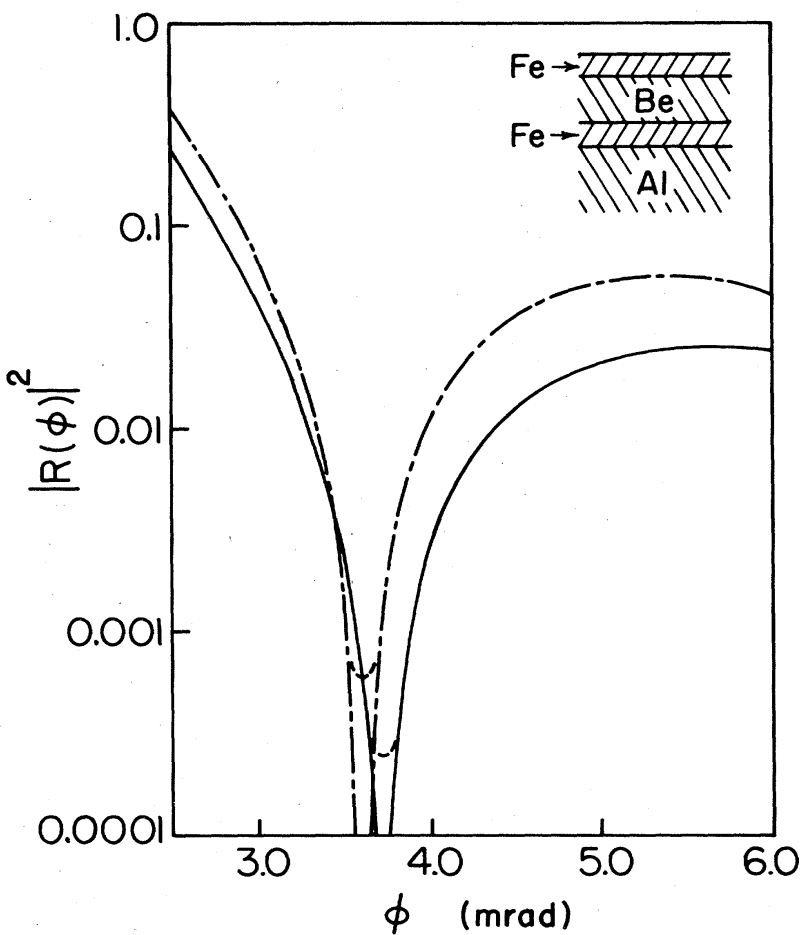

FIG. 6. Rocking curves for layered ultrathin films $(10-\AA$ $\mathrm{Fe}-64-\AA \mathrm{Be}-12-\AA \mathrm{Fe}-\mathrm{Al}$ ) (solid curve) and (15- $\mathrm{Ae}-64-\AA$ $\mathrm{Be}-17-\AA \mathrm{Fe}-\mathrm{Al}$ ) (dashed curve), reflecting 14.4-keV radiation.

For $\phi \simeq \phi_{c 2}(\mathrm{Fe}), R_{01}$ is small and positive and $F\left(l_{2}\right)$, as given by Eq. (5), is negative. To get destructive interference the thickness of film 1 should then be taken so that $2 g_{1} l_{1}=3 \pi / 2$, which requires $l_{1}$ to be a $3 \lambda / 8$ coated film,

$$
l_{1}=3 \lambda / 8\left(\phi^{2}-\phi_{c 1}^{2}\right)^{1 / 2} \text {. }
$$

For optimum suppression, the thickness of the UT film should be chosen to give an impedance match, i.e., $-F\left(l_{2}\right)=R_{01}$, which requires

$$
l_{2} \simeq \lambda r_{01} / \pi \phi_{c 2} \text {. }
$$

With $\mathrm{Al}\left(\phi_{c}=2.27 \mathrm{mrad}\right)$ as the backing and a $3 \lambda / 8$ coating, then at $\phi=\phi_{c 2}=3.84 \mathrm{mrad}$ these estimates give $l_{1}=104 \AA(\mathrm{Al})$ and $l_{2}=8 \AA(\mathrm{Fe})$. An exact procedure for determining the optimum $l_{1}, l_{2}$ is given in the following section.

\section{MULTILAYER GIAR FILMS}

The layered ultrathin GIAR films discussed above are examples of multilayer techniques. In the optical and infrared regions, multilayer techniques are widely used to produce more effective antireflection filters and to greatly extend the choice of suitable materials. There is extensive literature on such multilayer techniques, ${ }^{2-4}$ all of which can be carried over directly to GIAR films. However, the effectiveness of many of these techniques is severely limited by strong photoabsorption.

For GIAR films there are three main advantages to multilayer coatings: First, with additional layers, it is 
possible to reduce the density change in adjacent films, thereby reducing the amplitude of the interface reflections. Just as for the UT-GIAR films, interference is then between small amplitudes, which improves the effectiveness of the filter. Secondly, with more than one layer, it is possible to produce a secondary minimum close to the primary minimum, with the result that there is a greatly increased region of strong suppression. (In contrast, for a single film, the minima are regularly spaced at $2 \pi$ intervals of the phase $\psi_{1}=2 g_{1} l_{1}$, with intermediate regions of strong constructive interference.) The most important advantage is that with more than one layer, the thicknesses of the additional layers are new variable parameters and it is possible to achieve impedance match and phase cancellation by adjusting the film thicknesses. Such "thickness tuning" makes it possible to arbitrarily specify the incidence angle $\phi_{0}$, which is not possible for single-layer GIAR films and greatly extends the choice of film materials.

\section{A. Fractional wavelength multilayer films}

In the optical region, there are a number of filter techniques utilizing fractional wavelength coatings-primarily various combinations of half- and quarter-wave coatings. In these systems impedance matching is made by a proper choice of the indices of refraction of the various layers, i.e., by "index-of-refraction tuning." The advantage here is that for a nonabsorbing dielectric film, the characteristic matrix $\widetilde{\mathscr{M}}$ (see Appendix B of paper I) greatly simplifies for a quarter- or half-wave thickness and there is a consequent simplification of the analysis for a series of such films. However, in the presence of strong absorption, there is no special simplification at half- or quarterwavelengths and, hence, for GIAR films there is no particular advantage in using fractional wavelength films. Because of the limited usefulness, we will not give a complete account of fractional wavelength GIAR films, but we mention two cases of interest.

\section{Quarter-coated $\lambda / 2$ film}

As discussed in Sec. II, it is impossible to achieve an impedance match in a single half-wave film: the amplitude of the lower interface reflection is reduced by the substrate and by strong photoabsorption in transversing the film, leaving the wave too weak to cancel the upper surface reflection. However, the upper surface reflection can be reduced by coating the $\lambda / 2$ film with a low-density quarter-wave coat; for example, coating a $185-\AA \mathrm{Fe}$ film, which is a $\lambda / 2$ film for $14.4-\mathrm{keV}$ radiation at $\phi_{0}=4.5$ mrad, with a $55-\AA \mathrm{Al}$ film, which is a $\lambda / 4$ film at $\phi_{0}$. The addition of $\mathrm{Al}$ reduces the upper $\mathrm{Fe}$ interface reflection, and because of the $\lambda / 4$ condition, the $\mathrm{Al}-\mathrm{Fe}$ reflection is further reduced by destructive interference with the reflection from the upper Al surface.

In the single scattering approximation, the total reflection amplitude at $\phi_{0}$ is then given approximately by

$$
R\left(\phi_{0}\right) \simeq 2 R_{01}-R_{02}^{\dagger},
$$

where $R_{01}$ is the reflection amplitude for a single

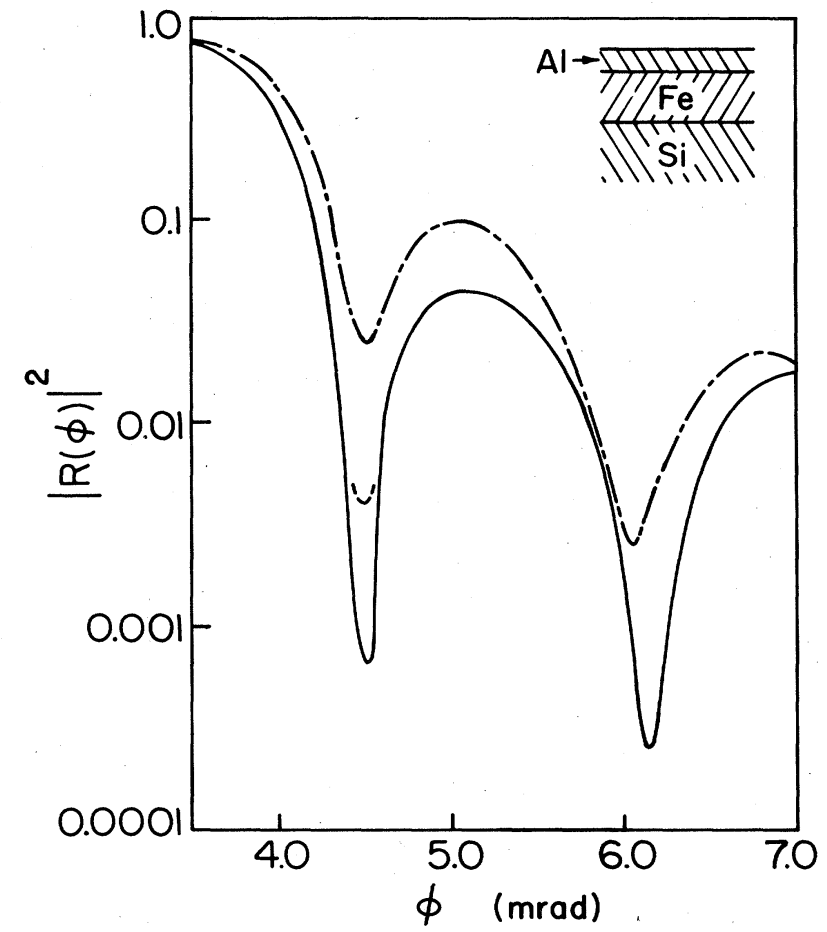

FIG. 7. Rocking curve for $\lambda / 2$ film of $\mathrm{Fe}\left(l_{2}=185 \AA\right)$ coated with a $\lambda / 4$ film of $\mathrm{Al}\left(l_{1}=55 \AA\right)$ with minimum at $\phi_{0}=4.5$ mrad. The dashed curve gives the uncoated response.

(vacuum-) Al surface and $R_{02}^{\dagger}$ is the total reflection amplitude for the $\lambda / 2$ film plus substrate, given approximately by Eq. (2). For an optimum coating, the quarterwave film should be chosen so that

$$
R_{01} \gtrsim \frac{1}{2} R_{02}^{\dagger}
$$

The main problems are finding a material of proper electron density, and the increased difficulty of achieving precise thickness control for two films rather than one.

For the $185-\AA \mathrm{Fe}$ film on $\mathrm{Si}, R_{02}^{\dagger} \simeq 0.16$ at $\phi_{0}=4.5$ mrad, while for A1, $2 R_{01}\left(\phi_{0}\right) \simeq 0.14$, a little low for an optimum coating. Nevertheless, as shown in Fig. 7 , the minimum reflectivity is reduced to $|R|^{2} \approx 7 \times 10^{-4}$ or $\left\langle|R|^{2}\right\rangle \approx 4 \times 10^{-3}$ when averaged over an angular spread of $\Delta \phi \approx 0.2 \mathrm{mrad}$, which is a significant improvement over the response for the uncoated film shown by the dashed curve.

\section{Impedance-matched $\lambda / 2 N-G I A R$ film}

Another multilayer technique, which illustrates several of the general features mentioned above but appears of limited practical use, is to coat a high-density mirror with a "staircase" series of $N-1$ films, each of optical path $\lambda / 2 N$ and with increasing electron density, to give equal reflection amplitudes at each interface, i.e., $R_{n, n+1}=R_{01}$ for $n=0,1, \ldots, N-1$.

For GIAR films, the impedance-match condition reduces to $\beta_{n+1} / \beta_{n}=\beta_{N}^{1 / N}$ or $\beta_{n}=\beta_{N}^{n / N}$, and the ampliude of each interface reflection is then 


$$
R_{n, n+1}=R_{01}=\frac{1-\beta_{N}^{1 / N}}{1+\beta_{N}^{1 / N}},
$$

which is strongly reduced from the uncoated mirror reflection amplitude of $\left(1-\beta_{N}\right) /\left(1+\beta_{N}\right)$. In the single scattering approximation the total scattering amplitude for the series is then

$$
R=\sum_{n=0}^{N-1} R_{n, n+1} \exp \left[i \sum_{m=0}^{n} \psi_{m}\right],
$$

where $\psi_{m}=2 g_{m} l_{m}=4 \pi \phi \beta_{m} l_{m} / \lambda$ is the phase for transversal through the $m$ th film.

The general phasor addition diagram for graphically summing the series of Eq. (13) is shown in Fig. 8(a). Here, $N=3$, and it is assumed $R_{n, n+1}$ is real and positive, i.e., increasing density, $\phi>\phi_{c}$, and $\sigma_{e}=0$.

With impedance-matched amplitudes $r_{n, n+1}$ the condition for destructive interference is then that all $\psi_{m}=2 \pi / N$ (or an integral multiple $\neq N$ ), which closes the phasor diagram as shown in Fig. 8(b). Each film will then be a " $\lambda / 2 N$ film," with the thickness of the $n$th film being

$$
l_{n}=\lambda /\left[2 N \phi_{0} \beta_{n}\left(\phi_{0}\right)\right] \text {. }
$$

The chosen angle $\phi_{0}$ is then the primary interference minimum. However, the $\psi_{m}$ are to first approximation linear in $\phi$ and, hence, as $\phi$ increases there will be secondary minima whenever all $\psi_{m}$ are approximately integral

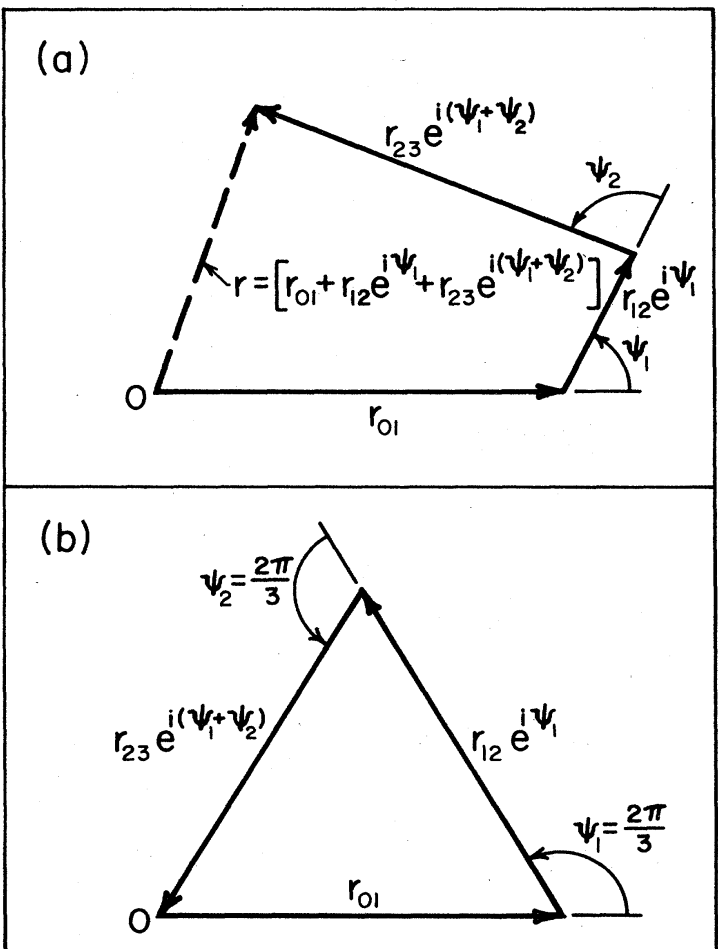

FIG. 8. (a) General phasor-addition diagram for three interface reflection amplitudes. (b) Closed phasor diagram for reflection from a mirror coated with two-impedance-matched $\left(r_{01}=r_{12}=r_{23}\right) \lambda / 6$ films $\left(\psi_{1}=\psi_{2}=2 \pi / 3\right)$. multiples of $2 \pi / N$.

An important point is that in the regions between the interference minima, the phasors are no longer perfectly constructive. This is in contrast to the single-film case which only involves two phasors $R_{01}$ and $R_{12} \exp \left(i \psi_{1}\right)$ which necessarily add constructively between any two minima. Hence, for the multilayer case, the intermediate maxima tend to be less pronounced. A basic problem here of course is finding a series of materials of proper electron density to satisfy the impedance-match condition and, in practice, the condition can only be approximately satisfied.

\section{B. Thickness-tuned multilayer GIAR films}

A much more powerful technique, and the main advantage of multilayer GIAR films, is the use of "thickness tuning," treating the film thicknesses $l_{1}, l_{2}, \ldots$ as parameters which can be adjusted to achieve both impedance match and phase cancellation. This is a distinct advantage over index-of-refraction tuning because, in contrast to electron densities which are fixed, film thicknesses can be varied continuously.

\section{Two films}

For a mirror coated with two films, the scattering amplitude in the single scattering approximation is

$$
R=R_{01}+R_{12} e^{i \psi_{1}}+R_{23} e^{i\left(\psi_{1}+\psi_{2}\right)},
$$

where as before, $\psi_{j}=\psi_{j}^{\prime}+i \psi_{j}^{\prime \prime}=2 g_{j} l_{j}=4 \pi \phi \beta_{j} l_{j} / \lambda$ is the complex phase shift for transversal through the $j$ th film, and $R_{n, n+1}=\left(\beta_{n}-\beta_{n+1}\right) /\left(\beta_{n}+\beta_{n+1}\right)$ is the reflection amplitude at the $n, n+1$ interface. If the film materials are chosen such that the combined magnitude of any two reflections exceeds that of the remaining surface, i.e., if

$$
r_{i j}<r_{k l}+r_{m n}
$$

then complete destructive interference can be achieved with the proper choice of the film thicknesses $l_{1}$ and $l_{2}$.

A simple graphical solution commonly used in the optical theory ${ }^{2}$ is given in Fig. 9 for the particular case of an Fe mirror coated with Sn (film 2) and Se (film 1). We are free to specify any $\phi_{0}$, as long as the reflection amplitudes satisfy the criteria equation (16). To be specific, we take $\phi_{0}=4.5 \mathrm{mrad}$, in which case $r_{01}=0.130, r_{12}=0.091$, and $r_{23}=0.103$ (all in the limit $\sigma_{e}=0$ ). In Fig. 10(a) the circle $C_{1}$ of radius $r_{12}$ is centered at the tip $P_{1}$ of the $R_{01}$ vector, the circle $C_{2}$ of radius $r_{23}$ is centered at the origin $P_{0}$, and the intersections $P_{2}, P_{2}^{\prime}$ determine the two possible solutions for completing the phasor diagram to give exact cancellation. For the solution $P_{2}$ of Fig. 9(b), $\psi_{1}=2.24$ rad and $\psi_{2}=1.70 \mathrm{rad}$, which determines the thicknesses to be $l_{1}=44 \AA(\mathrm{Se})$ and $l_{2}=40 \AA(\mathrm{Sn})$. For the second solution $P_{2}^{\prime}$ of Fig. $10(\mathrm{c})$, the film thicknesses are $l_{1}=80 \AA$ and $l_{2}=109 \mathrm{~A}$.

In this example, all $\boldsymbol{R}_{n, n+1}$ are real and positive, which implies increasing electron density, $\phi>\phi_{c 3}$, and $\sigma_{e}=0$. The graphical solution technique is easily modified to allow for negative $\boldsymbol{R}_{n, n+1}$ or more generally complex amplitudes, but it is still necessary to require that the phases $\psi_{1}$ 


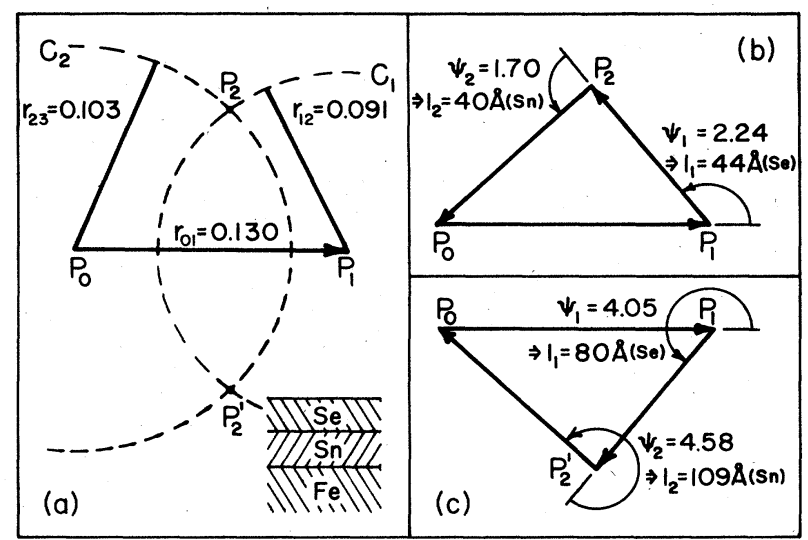

FIG. 9. Graphical solution for $l_{1}(\mathrm{Se})$ and $l_{2}(\mathrm{Sn})$ to give twofilm antireflection coat on $\mathrm{Fe}$ mirror to suppress the reflection of $14.4-\mathrm{keV}$ radiation incident at $\phi_{0}=4.5 \mathrm{mrad}$.

and $\psi_{2}$ be purely real, which is equivalent to the restriction $\phi>\phi_{c}$ and $\sigma_{e}=0$. Furthermore, the graphical solution is a single scattering approximation which ignores multiple reflections.

However, it is easy to give a completely general exact solution which takes into account multiple scattering and photoabsorption and which can be applied to any $\phi$ region, including $\phi<\phi_{c}$. This procedure is a direct modification of the general single-film solution given in paper $\mathbf{I}$ : For a mirror coated with two films, the exact reflection amplitude is

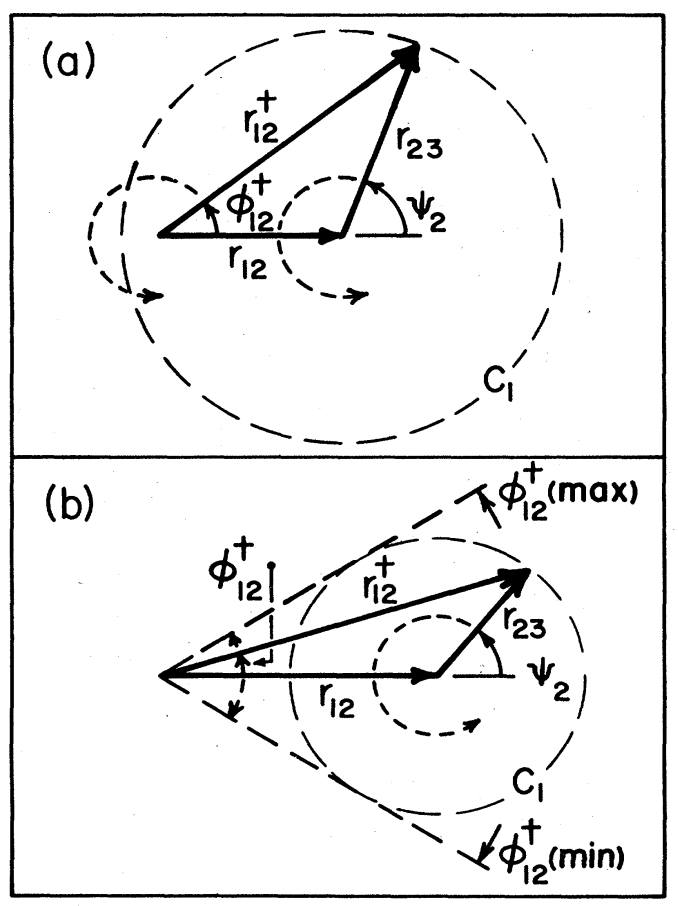

FIG. 10. Variation of $\phi_{12}^{\dagger}$ vs $\psi_{2}\left(=2 g_{2} l_{2}\right)$ for (a) $r_{23}>r_{12}$ and (b) $r_{23}<r_{12}$.

$$
\begin{aligned}
R & =\frac{R_{01}+R_{12}^{\dagger}\left(l_{2}\right) e^{i 2 g_{1} l_{1}}}{1+R_{01} R_{12}^{\dagger} e^{i 2 g_{1} l_{1}}} \\
& =\frac{r_{01} e^{i \phi_{01}}+r_{12}^{\dagger}\left(l_{2}\right) e^{-2 g_{1}^{\prime \prime} l_{1}} e^{i\left(\phi_{12}^{\dagger}\left(l_{2}\right)+2 g_{1}^{\prime} l_{1}\right)}}{1+R_{01} R_{12}^{\dagger} e^{i 2 g_{1} l_{1}}},
\end{aligned}
$$

where $R_{12}^{\dagger}\left(l_{2}\right)$ is the total reflection amplitude of film 2 plus substrate reflecting into medium 1 ,

$$
R_{12}^{\dagger}\left(l_{2}\right)=\frac{R_{12}+R_{23} e^{i 2 g_{2} l_{2}}}{1+R_{12} R_{23} e^{i 2 g_{1} l_{2}}} \equiv r_{12}^{\dagger}\left(l_{2}\right) e^{i \phi_{12}^{\dagger}\left(l_{2}\right)} .
$$

We have also introduced the notation for the amplitudes and phases of the individual interface reflection amplitudes as in Eq. (18) of paper I, $R_{i j}=r_{i j} \exp \left(i \phi_{i j}\right)$. Now, $R_{12}^{\dagger}\left(l_{2}\right)$ is an explicit function of $l_{2}$, so the amplitude and phase of the combined reflection can be "tuned" by varying the thickness $l_{2}$. From Eq. (17), the general impedance-match condition is then

$$
r_{01}=r_{12}^{\dagger}\left(l_{2}\right) e^{-2 g_{1}{ }^{\prime \prime} l_{1}} .
$$

Just as in Eq. (19) of paper I, this can be viewed as an equation for $l_{1}^{(=)}\left(l_{2}\right)$, the thickness film 1 must be taken to give an impedance match,

$$
l_{1}^{(=)}\left(l_{2}\right)=\frac{\ln \left(r_{12}^{\dagger}\left(l_{2}\right) / r_{01}\right)}{2 g_{1}^{\prime \prime}} .
$$

As a function of $l_{2}, r_{12}^{\dagger}\left(l_{2}\right)$ and, hence, $l_{1}^{i=)}\left(l_{2}\right)$ are oscillatory but slowly damped to a constant value in the limit of very large $l_{2}$. Since $\left|r_{12}-r_{23}\right|<r_{01}<\left[r_{12}+r_{23}\right]$, then $l_{1}^{(=)}\left(l_{2}\right)$ oscillates positive to negative.

The condition for destructive interference is

$$
\exp \left\{i\left[2 g_{1}^{\prime} l_{1}+\phi_{12}^{\dagger}\left(l_{2}\right)-\phi_{01}\right]\right\}=-1,
$$

which requires the relative phase to be an odd multiple of $\pi$. For a given $l_{2}$, the thickness of film 1 necessary to give a $(2 n+1) \pi$ relative phase must then be

$$
l_{1}^{(2 n+1) \pi}\left(l_{2}\right)=\frac{(2 n+1) \pi-\phi_{12}^{\dagger}\left(l_{2}\right)+\phi_{01}}{2 g_{1}^{\prime}} .
$$

If $r_{12}<r_{23}$, then $\phi_{12}^{\dagger}\left(l_{2}\right)$ monotonically increases with $l_{2}$, as shown by the phasor diagram, Fig. 10(a), and in this case, $l_{1}^{(2 n+1) \pi}$ decreases monotonically to zero. On the other hand, if $r_{12}>r_{23}$, then $\phi_{12}^{\ddagger}\left(l_{2}\right)$ and, hence, $l_{1}^{(2 n+1) \pi}\left(l_{2}\right)$ is an oscillatory function of $l_{2}$, as indicated in Fig. 10(b).

The necessary thicknesses $l_{1}, l_{2}$ for antireflection coatings are then determined by the intersections of the $l_{1}^{(=)}\left(l_{2}\right)$ curves for impedance match with the $l_{1}^{(2 n+1) \pi}\left(l_{2}\right)^{\prime}$ curves for destructive interference.

In Fig. 11 we give $l_{1}^{(=)}, l_{1}^{\pi}\left(l_{2}\right)$, and $l_{1}^{3 \pi}\left(l_{2}\right)$ for a Fe mirror coated with Sn (film 2) and Se (film 1), with $\phi_{0}$ chosen as $4.5 \mathrm{mrad}$. The intersections $P_{2}, P_{2}^{\prime}$ give film thickness $l_{1}, l_{2}$ close to the phasor diagram solutions but shifted somewhat due to the effects of multiple reflections and photoabsorption.

The Fe mirror should then be coated with $l_{1}=44 \AA$ of 


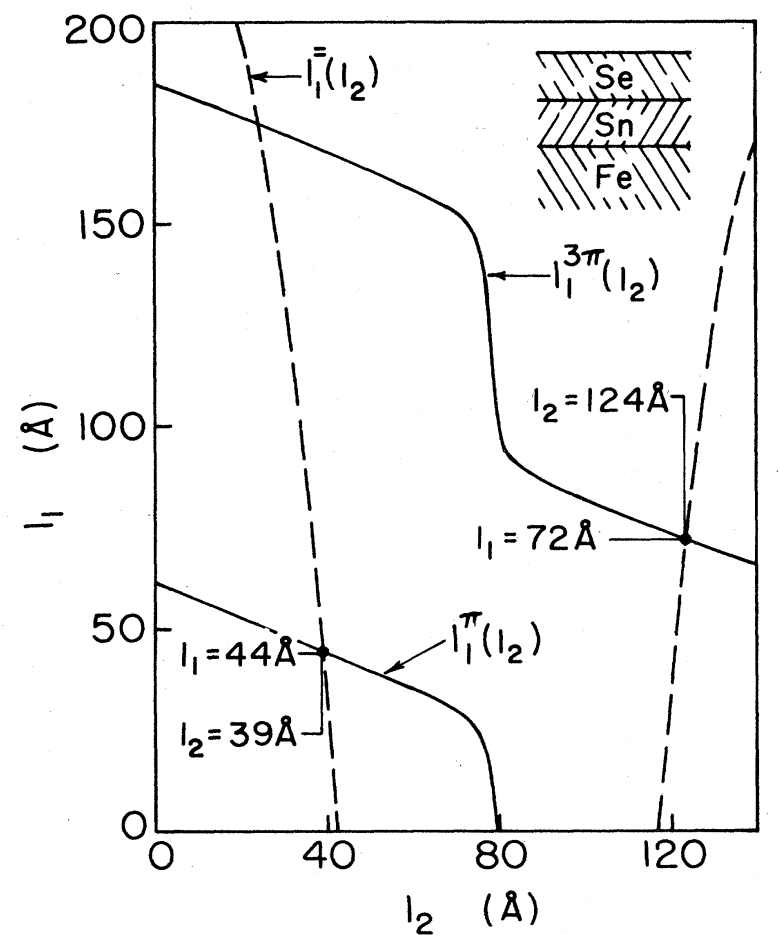

FIG. 11. $l_{1}^{(=)}\left(l_{2}\right), l_{1}^{\pi}\left(l_{2}\right)$, and $l_{1}^{3 \pi}\left(l_{2}\right)$ for Fe mirror coated with Sn (film 2) and Se (film 1), with $\phi_{0}$ chosen as $4.5 \mathrm{mrad}$. The intersections $l_{1}(\mathrm{Se})$ and $l_{2}(\mathrm{Sn})$ give antireflection solutions.

Se and $l_{2}=39 \AA$ of Sn to give a multilayer GIAR film with primary minimum at $\phi_{0}=4.5 \mathrm{mrad}$ when reflecting 14.4-keV radiation. Figure 12 gives the rocking curve for this case, with the dashed curve at the minimum giving the reflectivity averaged over an angular spread of $\Delta \phi=0.2 \mathrm{mrad}$. We note in particular that the angular region of strong suppression is somewhat broader than for the single-layer GIAR films and the first subsidiary max-

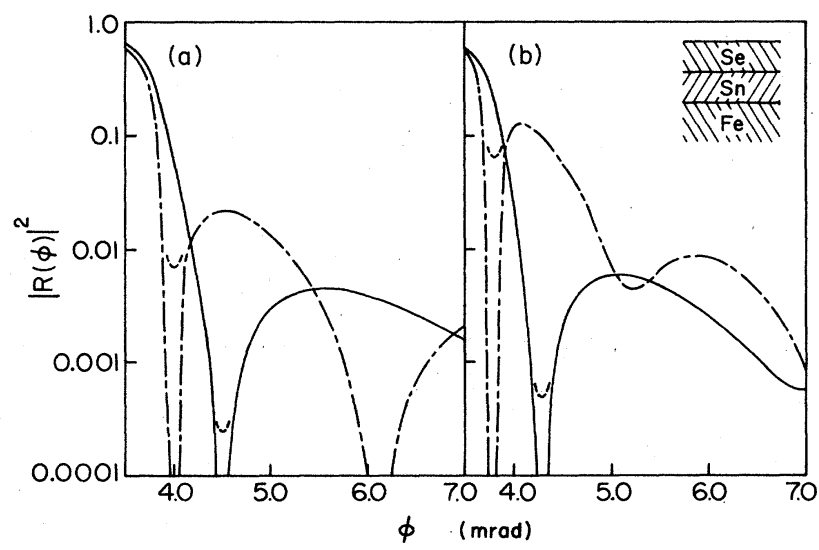

FIG. 12. Rocking curves $|R|^{2}$ vs $\phi$ for different coatings $l_{1}(\mathrm{Se})$ and $l_{2}(\mathrm{Sn})$ on $\mathrm{Fe}$, determined to give antireflection coats at $\phi_{0}=4.5 \mathrm{mrad}\left(l_{1}=44 \AA, l_{2}=39 \AA\right), \phi_{0}=4.3 \quad\left(l_{1}=45 \AA\right.$, $\left.l_{2}=51 \AA\right), \phi_{0}=4.0\left(l_{1}=39 \AA, l_{2}=86 \AA\right)$, and $\phi_{0}=3.8\left(l_{1}=19 \AA\right.$, $l_{2}=155 \AA$ ). imum is much less pronounced [compare, for example, Fig. 10(a) of paper I], in agreement with the previous qualitative discussion.

Also in Fig. 12, we give the optimum thicknesses $l_{1}(\mathrm{Se})$ and $l_{2}(\mathrm{Sn})$ and the 14.4-keV rocking curves for $\mathrm{Fe}$ mirrors coated with multilayer GIAR films, with primary minima chosen to occur at $\phi_{0}=4.3,4.0$, and $3.8 \mathrm{mrad}$, respectively. For the last case, $\phi_{0}<\phi_{c}(\mathrm{Fe})$ and the response is similar to the damping-stabilized films of Sec. III of paper I.

One of the important features of thickness-tuned multilayer GIAR films is that they greatly expand the range of possible materials for producing GIAR films-the only restriction which must be met is Eq. (16). With different choices of materials the response can be quite different.

An example of particular interest is a $\mathrm{Fe}$ mirror $\left(\phi_{c 3}=3.84 \mathrm{mrad}\right)$ coated with $\mathrm{Sn}\left(\phi_{c 2}=3.45 \mathrm{mrad}\right)$ and $\mathrm{Al}$ $\left(\phi_{c l}=2.27 \mathrm{mrad}\right)$. Figure 13 gives the curves $l_{1}^{(=)}\left(l_{2}\right)$, $l_{1}^{\pi}\left(l_{2}\right)$, and $l_{1}^{3 \pi}\left(l_{2}\right)$ for $\phi_{0}=4.5 \mathrm{mrad}$. For the lowest thickness solution, $l_{1}=47 \AA$ ( $(\mathrm{Al}), l_{2}=66 \AA(\mathrm{Sn})$ and for the second solution, $l_{1}=66 \AA$ (Al), $l_{2}=87 \AA$ (Sn). Figure 14 gives the rocking curve for the first solution and shows several interesting features: First, the primary minimum is very deep and broad, with an average reflectivity over $0.2 \mathrm{mrad}$ of $\left\langle|R|^{2}\right\rangle \simeq 1.1 \times 10^{-4}$. Furthermore, the first secondary minimum is now quite close, occurring at 5.6 $\mathrm{mrad}$, the intermediate maximum at $5.0 \mathrm{mrad}$ only peaks to $|R|^{2} \approx 7.5 \times 10^{-4}$ and the reflectivity is less than $10^{-3}$ over the exceptionally broad angular region of $1.7 \mathrm{mrad}$.

The reason for this behavior can be understood qualitatively from the phasor diagrams given in Fig. 15: Figure

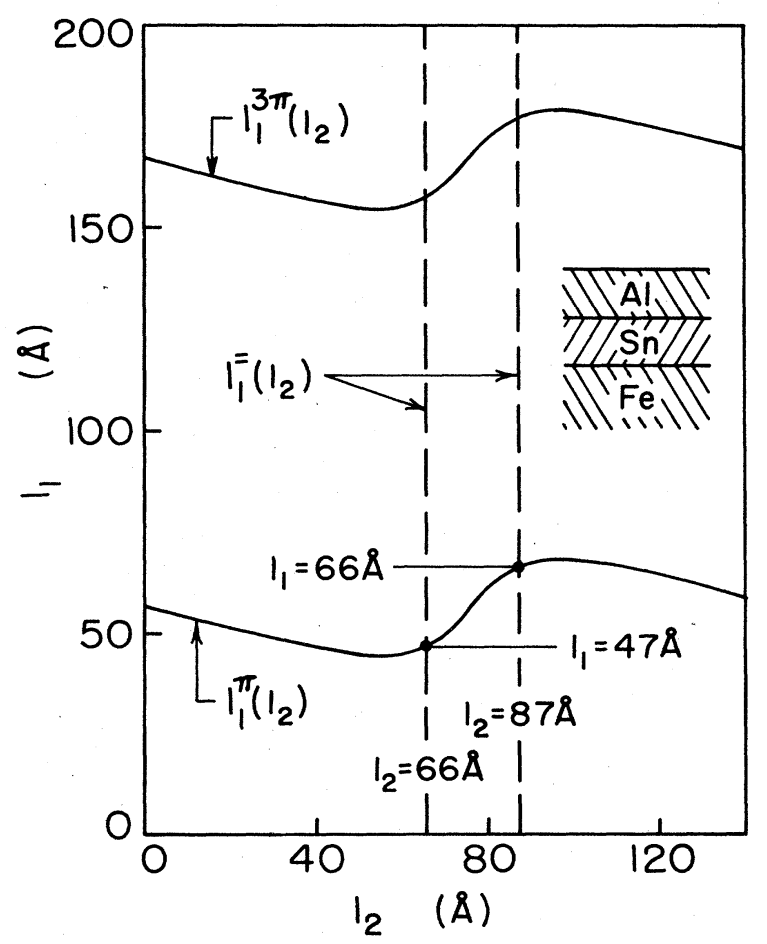

FIG. 13. Graphical solution for $l_{1}(\mathrm{Al})$ and $l_{2}(\mathrm{Sn})$ for antireflection coatings on an $\mathrm{Fe}$ mirror, reflecting 14.4-keV radiation at $\phi_{0}=4.5 \mathrm{mrad}$. 


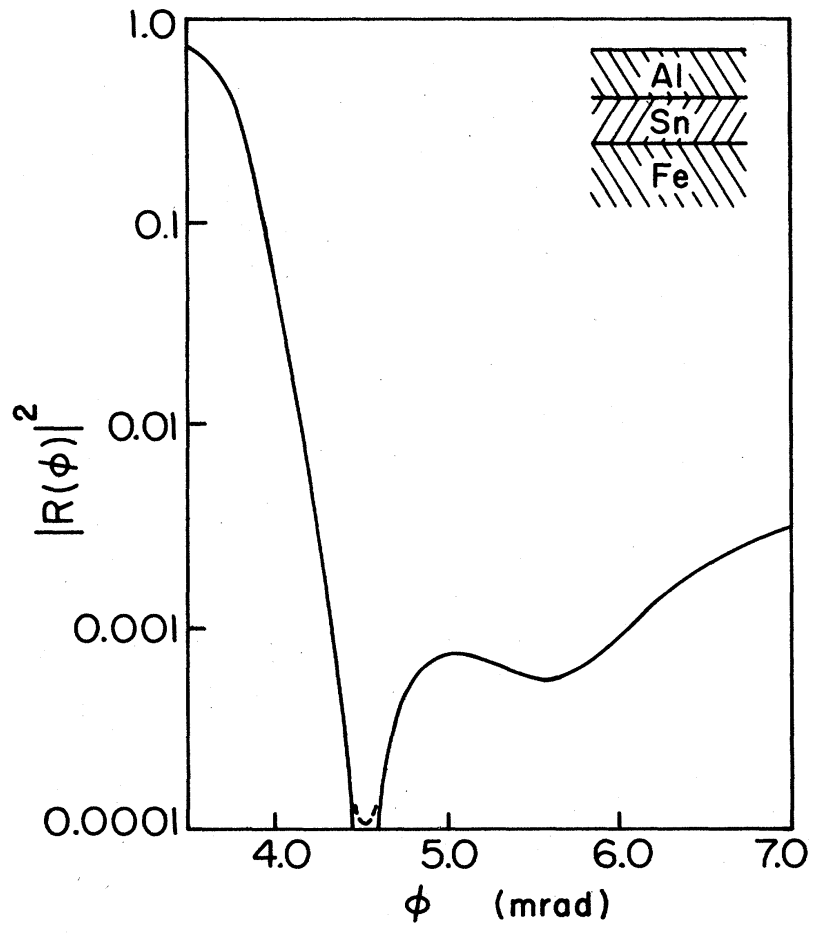

FIG. 14. Rocking curve $|R|^{2}$ vs $\phi$ for Fe mirror coated with $l_{1}=47 \AA$ (Al), $l_{2}=66 \AA(\mathrm{Sn})$, reflecting $14.4-\mathrm{keV}$ radiation.

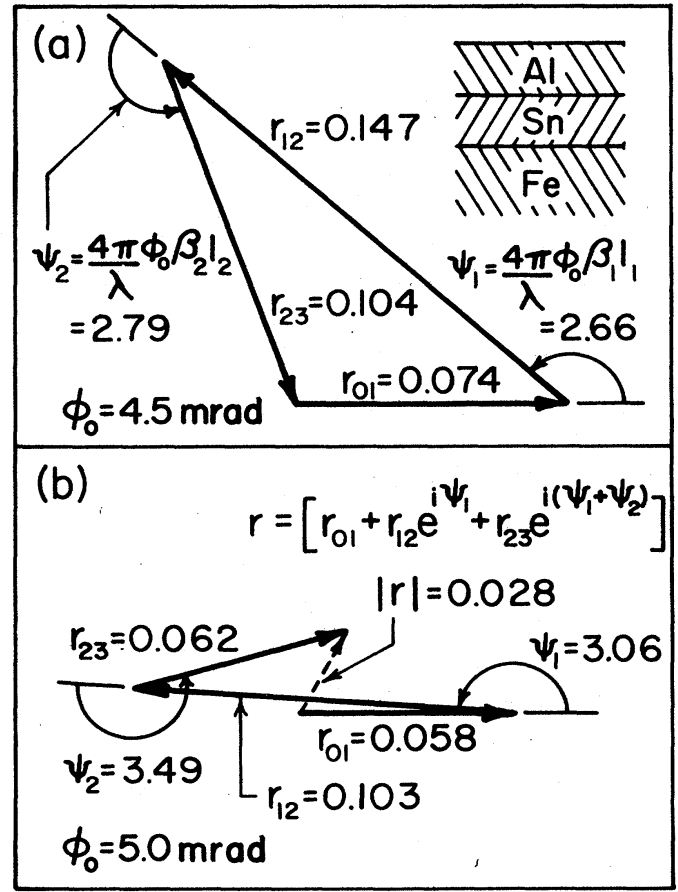

FIG. 15. Phasor-addition diagram for the reflection amplitude $R$ for the mirror of Fig. 14, at (a) the primary minimum $\phi_{0}=4.5 \mathrm{mrad}$, and at (b) the first secondary maximum $\phi=5.0$ mrad. 15(a) gives the phasor diagram at the primary minimum $\phi_{0}=4.5 \mathrm{mrad}$. Because $\mathrm{Al}$ is low density while both $\mathrm{Sn}$ and $\mathrm{Fe}$ have high electron densities, the largest reflection amplitude is $r_{12}$ at the Al-Sn interface and the phasor triangle is strongly "tilted" to the left. Now, as $\phi$ increases, $\psi_{1}$ increases, and the effect is to rotate the tip of the $R_{23}$ phasor downward, which is also the tip of the total $R$. However, $\psi_{2}$ also increases, which tends to rotate the tip back up towards the first quadrant. The combination of these two counteracting rotations, plus the fact that the lengths of the phasor vectors decrease with increasing $\phi$, work to keep the resultant $R$ small over an exceptionally large angular region. Figure 15(b) shows the resulting phasor addition diagrams at $\phi=5.0$ (first secondary maximum).

Finally, the two-film formalism can also be applied to give an exact solution for the $3 \lambda / 8$-coated UT film discussed in the preceding section. We can in fact generalize, letting film 1 and substrate 3 be any two low-density media (but if not identical, the coating will not be a $3 \lambda / 8$ coating) and we need not require $\phi \simeq \phi_{c 2}$. As a particular example, we give the results for an Al-Fe-Si series. Choosing the primary minimum to occur at $\phi_{0}=\phi_{c 2}(\mathrm{Fe})=3.84 \mathrm{mrad}$, the optimum thicknesses are $l_{1}=106 \AA(\mathrm{Al})$ and $l_{2}=10 \AA(\mathrm{Fe})$, while at $\phi_{0}=4.5 \mathrm{mrad}$, $l_{1}=83 \AA$ and $l_{2}=8 \AA$. The corresponding rocking curves are shown in Fig. 16.

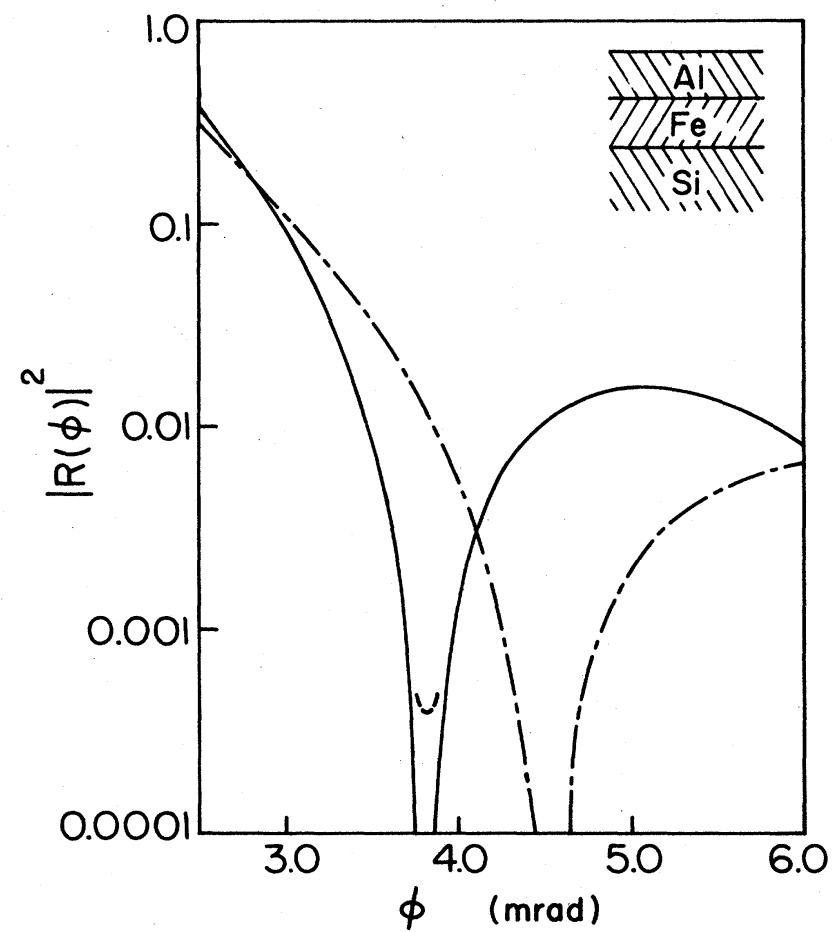

FIG. 16. Rocking curves $|R|^{2}$ vs $\phi$ for reflection of 14.4$\mathrm{keV}$ radiation from $\mathrm{Si}$ mirrors coated with an ultrathin film $l_{2}(\mathrm{Fe})$ which is coated with a low-density film $l_{1}(\mathrm{Al})$. Here, $l_{1}$ and $l_{2}$ have been determined to give a minimum at $\phi_{0}=\phi_{c}$ $(\mathrm{Fe})=3.84\left(l_{1}=106 \AA, l_{2}=10 \AA\right)$ and at $\phi_{0}=4.5 \mathrm{mrad}\left(l_{1}=83\right.$ $\AA, l_{2}=8 \AA$ ). This is a generalization of a $3 \lambda / 8$-coated ultrathin film. 


\section{Three or more films}

Additional degrees of freedom are introduced by increasing the number of films. For a mirror coated with three films, the necessary condition for there to be antireflection solutions for some choice of $l_{1}, l_{2}$, and $l_{3}$ is that

$$
r_{i j}<r_{k l}+r_{m n}+r_{p q} .
$$

However, there will now be a continuum of possible solutions rather than just two. This is easily seen from the graphical construction shown in Fig. 17 for the specific case of an $\mathrm{Al}(1)-\mathrm{Se}(2)-\mathrm{Sn}(3)$ series coated on $\mathrm{Fe}(4)$ with $\phi_{0}=4.5 \mathrm{mrad}$ : the circle $C_{1}$ of radius $r_{12}$ is centered at the tip $P_{1}$ of the $R_{01}$ vector and the circle $C_{3}$ of radius $r_{34}$ is centered at the origin $P_{0}$. The circle $C_{2}$ of radius $r_{23}$ is centered at $P_{2}$, which can be any point on $C_{1}$. The intersections $P_{3}$ and $P_{3}^{\prime}$ of $C_{2}$ and $C_{3}$ determine the two possible solutions for completing the phasor diagram in order that it give exact cancellation. However, since $P_{2}$ can be selected arbitrarily on $C_{1}$, there is in fact a continuum of possible solutions.

In Fig. 17 we show solutions for two different choices of $P_{2}$. From the measured $\psi_{1}, \psi_{2}, \psi_{3}$, the appropriate film thicknesses are $l_{1}=28 \AA(\mathrm{Al}), l_{2}=19 \AA(\mathrm{Se})$, and $l_{3}=52$ $\AA$ (Sn) (for the solid-line vectors of the diagram) and $l_{1}=14 \AA, l_{2}=33 \AA$, and $l_{3}=44 \AA$ (for the dashed-line vectors).

The exact treatment is a direct extension of the twofilm case: For a mirror coated with three films, the reflection amplitude is

$$
\begin{aligned}
R & =\frac{R_{01}+R_{12}^{\dagger}\left(l_{2}, l_{3}\right) e^{i 2 g_{l} l_{1}}}{1+R_{01} R_{12}^{\dagger}\left(l_{2}, l_{3}\right) e^{i 2 g_{1} l_{1}}} \\
& =\frac{r_{01} e^{i \phi_{01}}+r_{12}^{\dagger}\left(l_{2}, l_{3}\right) e^{-2 g_{1}^{\prime \prime} l_{1}} e^{i\left(\phi^{\dagger}\left(l_{2}, l_{3}\right)+2 g_{1}^{\prime} l_{1}\right)}}{1+R_{01} R_{12}^{\dagger} e^{i 2 g_{1} l_{1}}}
\end{aligned}
$$

where now

$$
R_{12}^{\dagger}\left(l_{2}, l_{3}\right) \equiv r_{12}^{\dagger}\left(l_{2}, l_{3}\right) \exp \left[i \phi_{12}^{\dagger}\left(l_{2}, l_{3}\right)\right]
$$

is the compound reflection amplitude for films 2 and 3

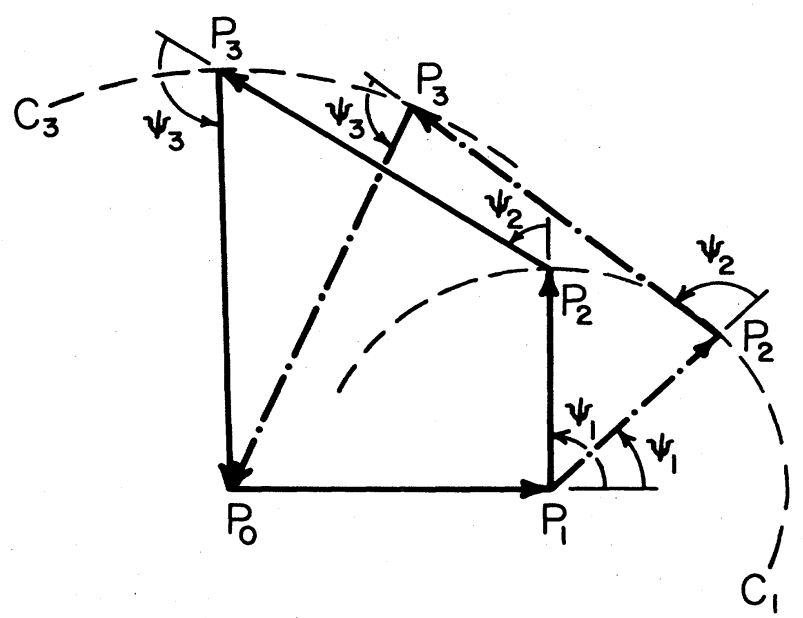

FIG. 17. Graphical solution for $l_{1}(\mathrm{Al}), l_{2}(\mathrm{Se})$, and $l_{3}(\mathrm{Sn})$.

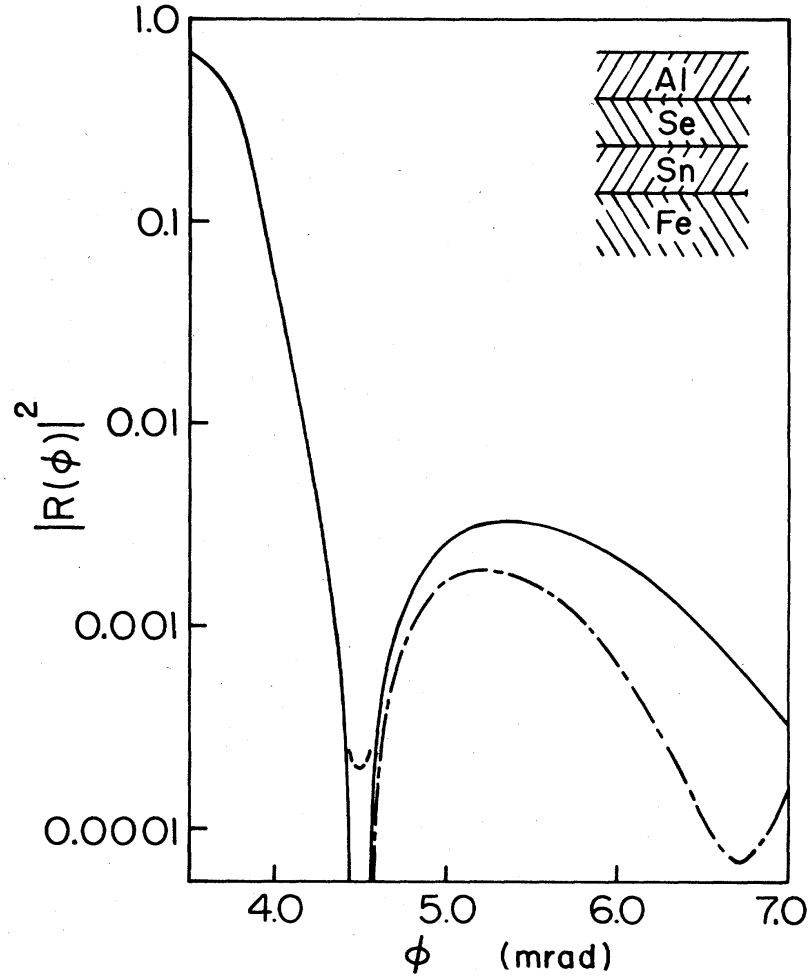

FIG. 18. Rocking curves $|R|^{2}$ vs $\phi$ for reflection of 14.4$\mathrm{keV}$ radiation from $\mathrm{Fe}$ mirrors coated with $l_{1}(\mathrm{Al}), l_{2}(\mathrm{Se})$, and $l_{3}(\mathrm{Sn}) . \quad l_{3}$ is set at (a) $44 \AA$ and (b) $52 \AA$, and $l_{1}$ and $l_{2}$ have been determined to give an antireflection minimum at $\phi_{0}=4.5 \mathrm{mrad}$. The solid curve is for $l_{1}=20 \AA, l_{2}=30 \AA$, and $l_{3}=44 \AA$, and the dashed curve is for $l_{1}=30 \AA, l_{2}=19 \AA$, and $l_{3}=52 \AA$.

plus substrate reflecting into medium 1 ,

$$
R_{12}^{\dagger}\left(l_{2}, l_{3}\right)=\frac{R_{12}+R_{23}^{\dagger}\left(l_{3}\right) e^{i 2 g_{2} l_{2}}}{1+R_{12} R_{23}^{\dagger}\left(l_{3}\right) e^{i 2 g_{2} l_{2}}}
$$

and $R_{23}^{\dagger}\left(l_{3}\right)$ is the compound reflective amplitude for film 3 plus substrate reflecting into medium 2 ,

$$
R_{23}^{\dagger}\left(l_{3}\right)=\frac{R_{23}+R_{34} e^{i 2 g_{3} l_{3}}}{1+R_{23} R_{34} e^{i 2 g_{3} l_{3}}} .
$$

We now arbitrarily fix either $l_{2}$ or $l_{3}$ in Eq. (24) [consistent with the restriction equation (23)]. The previous analysis then goes through with $r_{12}^{\dagger}\left(l_{2}, l_{3}\right)$ and $\phi_{12}^{\dagger}\left(l_{2}, l_{3}\right)$, replacing $r_{12}^{\dagger}\left(l_{2}\right)$ and $\phi_{12}^{\dagger}\left(l_{2}\right)$ in Eqs. (19)-(22). The extension to more than three films is made in the same manner.

TABLE I. Optimum thickness $l_{1}(\mathrm{Fe})$ and $l_{2}(\mathrm{Be})$ for several choices of $l_{3}(\mathrm{Fe})$.

\begin{tabular}{ccc}
\hline \hline $\begin{array}{c}l_{3}(\mathrm{Fe}) \\
(\AA)\end{array}$ & $l_{1}(\mathrm{Fe})$ & $l_{2}(\mathrm{Be})$ \\
$(\AA ̊ ̊)$ & $(\AA)$ \\
\hline 12 & $(10$ & 60 \\
17 & 15 & 57 \\
30 & 51 & 26 \\
\hline \hline
\end{tabular}




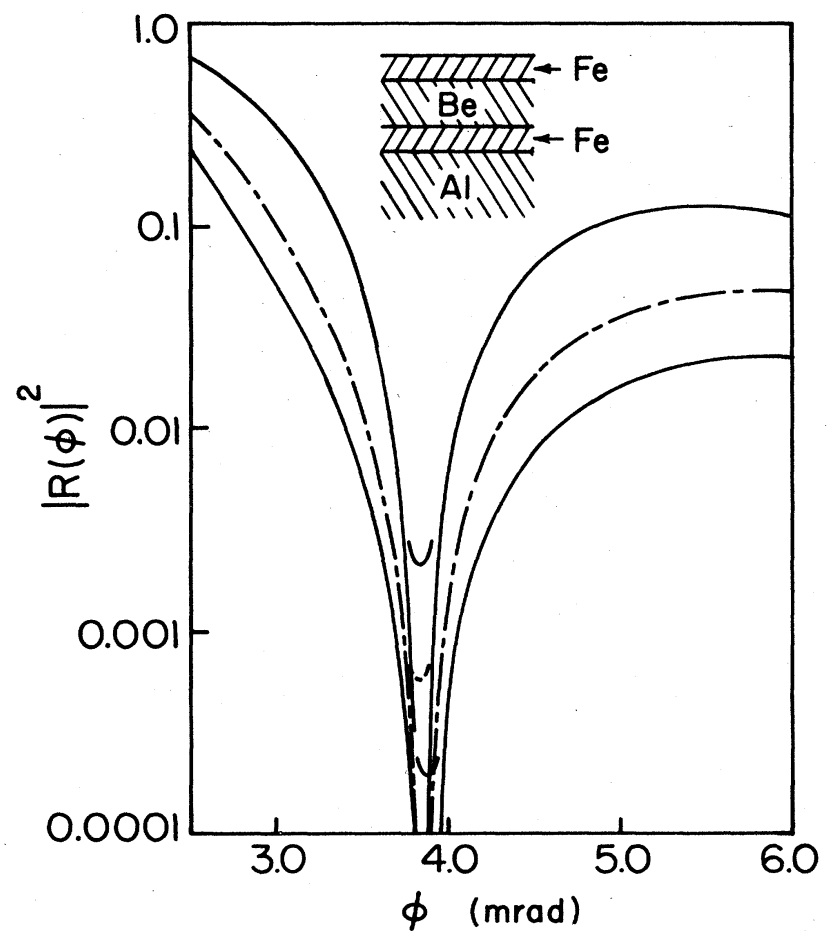

FIG. 19. Rocking curves for layered ultrathin films $l_{1}(\mathrm{Fe})$, $l_{2}(\mathrm{Be})$, and $l_{3}(\mathrm{Fe})$ on Al. Here, $l_{3}(\mathrm{Fe})$ has been set at (a) $12 \AA$, (b) $17 \AA$, and (c) $30 \AA$, and $l_{1}(\mathrm{Fe})$ and $l_{2}(\mathrm{Be})$ have been determined to give the antireflection minimum at $\phi_{0}=\phi_{c}(\mathrm{Fe})=3.84$ mrad. For the lower solid curve, $l_{1}=10 \AA, l_{2}=60 \AA$, and $l_{3}=12 \AA$; for the dashed curve, $l_{1}=15 \AA, l_{2}=57 \AA, l_{3}=17 \AA$; and for the upper solid curve, $l_{1}=26 \AA, l_{2}=51 \AA$, and $l_{3}=30$ Å.

For the previous example of Al-Se-Sn coated on $\mathrm{Fe}$, if we choose $\phi_{0}=4.5 \mathrm{mrad}$ and set $l_{3}=52 \AA(\mathrm{Sn})$, then the optimum thicknesses are $l_{1}=30 \AA(\mathrm{Al})$ and $l_{2}=19 \AA$ (Se), while if we set $l_{3}=44 \AA$ (Sn), then $l_{1}=20 \AA$ (Al) and $l_{2}=30 \AA$ (Se). In both cases, $l_{1}$ and $l_{2}$ are shifted from the graphical solutions by the effects of photoabsorption and multiple scattering. Figure 18 shows the rocking curves for the two cases. For either choice of parameters the primary minimum is at $\phi_{0}=4.5 \mathrm{mrad}$, but the locations of the secondary minima vary.

One particular application of the three-film formalism is to give optimum thicknesses $l_{1}, l_{2}, l_{3}$ for the layered UT-GIAR films discused in the previous section; for example, two UT layers of Fe separated by $\mathrm{Be}$ on an $\mathrm{Al}$ substrate. Table I gives the optimum thicknesses $l_{1}(\mathrm{Fe})$, and $l_{2}(\mathrm{Be})$ for several different choices of $l_{3}(\mathrm{Fe})$, determined to give the primary minimum at $\phi_{0}=\phi_{c 3}(\mathrm{Fe})=3.84 \mathrm{mrad}$. The resulting rocking curves are given in Fig. 19. More generally, the UT films 1 and 3 can be taken as any two high-density materials, media 2 and 4 can be any two low-density materials, and $\phi_{0}$ does not need to be restricted to be equal to $\phi_{c}$.

\section{TAPERED IMPEDANCE GIAR FILMS}

A limiting case of multilayer GIAR films is the coating of a mirror with a single "tapered" film in which the elec- tron density varies slowly from a very low value to the density of the mirror; e.g., an $\mathrm{Fe}$ mirror coated with a carbon-iron film $\mathrm{C}_{x} \mathrm{Fe}_{1-x}$, with the carbon fraction $x_{1}(z)$ slowly decreasing from $1 \rightarrow 0$ as $z \propto 0 \rightarrow l_{1}$. If we imagine the film as composed of a large number of strips $\Delta z$, there will be a reflection $R_{01}$ from the top surface (vacuumcarbon) and weak reflections,

$R_{z, z+\Delta z}=\left(\frac{\beta(z)-\beta(z+\Delta z)}{\beta(z)+\beta(z+\Delta z)}\right) \simeq-\frac{d \beta}{d z} \Delta z / 2 \beta(z)$,

at each interface. The total reflection can be calculated exactly from the multilayer formula, Eq. (12), of paper I or in the single scattering approximation as

$$
R \simeq R_{01}+\sum_{z=0}^{l_{1}} R_{z, z+\Delta z} \exp \left[i \int_{0}^{z} 2 g\left(z^{\prime}\right) d z^{\prime}\right] .
$$

The essential point is that by taking a slow gradient, the internal interface reflections are very weak and, if the film is taken sufficiently thick, the internal reflections will also tend to destructively interfere. The total reflection is then dominated by the top surface reflection $R_{01}$ (pure C) which is strongly reduced from a pure Fe reflection $R_{02}$. The degree of reduction depends on how the electron density is chosen to vary and on the film thickness $l_{1}$.

Near-total internal cancellation can be achieved by " $\lambda / N$ tapering:" For a chosen $\phi_{0}$ take " $\lambda / N$ increments" $\Delta z=\lambda /\left[N \phi_{0} \beta(z)\right]$ ( $N$ large and arbitrary) with the density increasing to give a constant reflection amplitude $R_{z, z+\Delta z}(\phi)=\alpha$ in Eq. (27). The resulting differential equation for $\beta(z)$, with the limits $\beta(z=0)=\beta_{1}\left(\phi_{0}\right)$, $\beta\left(z=l_{1}\right)=\beta_{2}\left(\phi_{0}\right)$, determines the required $\beta(z)$ as

$$
\beta(z)=\beta_{1} /\left[1+\left(z / l_{1}\right)\left(\beta_{1} / \beta_{2}-1\right)\right] .
$$

This also determines the required fractional concentration $x_{1}(z)$ of carbon, since $\beta(z)=\left[1-\phi_{c}^{2}(z) / \phi_{0}^{2}\right]^{1 / 2}$, and the critical angle for a two-component mixture is $\phi_{c}(z)=\left\{\left(x_{1}(z) \phi_{c 1}^{2}+\left[1-x_{1}(z)\right] \phi_{c 2}^{2}\right\}^{1 / 2}\right.$. With this choice of density variation, the total reflectivity at $\phi_{0}$ becomes (in the single scattering approximation)

$$
R\left(\phi_{0}\right) \simeq R_{01}-i \alpha^{\prime}\left(e^{i 2 k \phi_{0} \bar{\beta} l_{1}}-1\right),
$$

with

$$
\bar{\beta}=\left[\beta_{2} \beta_{1} /\left(\beta_{1}-\beta_{2}\right)\right] \ln \left(\beta_{1} / \beta_{2}\right)
$$

and

$$
\alpha^{\prime}=\left[\ln \left(\beta_{1} / \beta_{2}\right) / 4 k \phi_{0} \bar{\beta} l_{1}\right] .
$$

By tapering over a sufficiently large thickness $l_{1}$, then, $\alpha^{\prime} \lesssim R_{01}\left(\ll R_{02}\right)$ and the top surface reflection dominates. For $\mathrm{C}_{x} \mathrm{Fe}_{1-x}$ with $\phi_{0}=4.3 \mathrm{mrad}$, this requires $l_{1} \gtrsim 300$ $\AA$. However, it is also possible to achieve near-total destructive interference of the internal reflections by taking $l_{1} \simeq n\left(\lambda / 2 \phi_{0} \bar{\beta}\right)$, which for the $\mathrm{C}_{x} \mathrm{Fe}_{1-x}$ example requires $l_{1} \simeq n(160 \AA)$.

Figure 20 shows the rocking curves, calculated from Eq. (12) of paper I using $\Delta z=20 \AA$ strips, for an Fe mirror coated with a tapered $\mathrm{C}_{x} \mathrm{Fe}_{1-x}$ film, with the density 


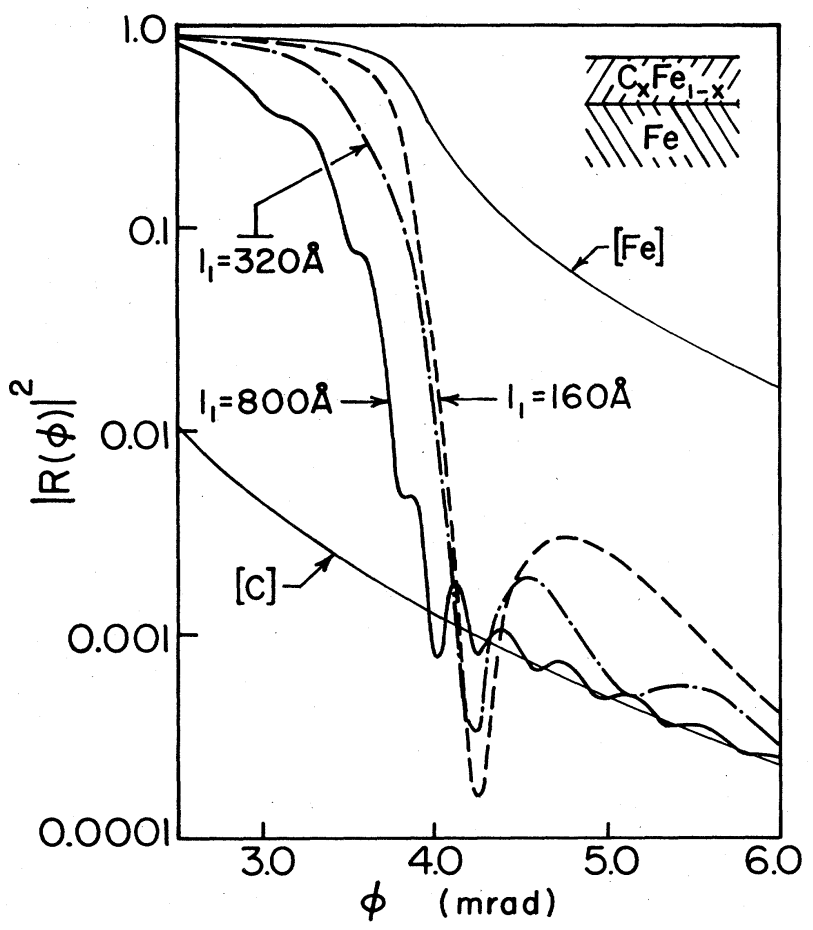

FIG. 20. Rocking curves for Fe mirror coated with tapered $\mathrm{C}_{x} \mathrm{Fe}_{1-x}$ film, with density variation $x(z)$ taken to produce a $\lambda / N$-tapered film at $\phi_{0}=4.3 \mathrm{mrad}$ for $l_{1}=160,320$, and $800 \AA$.

variation taken to produce a $\lambda / N$-tapered film at $\phi_{0}=4.3$ $\mathrm{mrad}$ for $l_{1}=160,320$, and $800 \mathrm{~A}$. The reflectivities $\left|R_{01}\right|^{2}$ and $\left|R_{02}\right|^{2}$ for pure $\mathrm{C}$ and $\mathrm{Fe}$ are also given for comparison. The interference minimum is slightly shifted from $\phi_{0}$ and, more importantly, there is a partial cancellation of the upper surface reflection so that the reduction is even stronger than anticipated by the single scattering approximation equation (30). Even away from $\phi_{0}$, where there are no exact phase cancellations, the reflectivity is still strongly reduced by the tapering, and for large angles approaches $\left|R_{01}\right|^{2}$.

The main advantage of such a tapered GIAR film is that it gives a strong suppression over a very broad angular range and, correspondingly, over a broad frequency range. The obvious disadvantage is the control problem of fabricating a film with the proper density variations. There are other equally good tapering schemes, and even stronger cancellation of the upper surface reflection should be possible.

\section{SUMMARY}

The purpose of this paper has been to examine more general techniques for producing grazing-incidence antireflection films to suppress the reflection of $1 \AA \mathrm{x}$ rays. Our main conclusions follow.

If a mirror is coated with a film of higher electron density, no antireflection films are possible; however, the reflectivity can still be reduced $\left(\approx \frac{1}{5}\right.$ times) by using a half-wave film interference minimum. Although the intensity reduction is much less pronounced than for a $\lambda / 4$-GIAR film, a $\lambda / 2$ film has the advantage that interference minima will occur for any chosen $l_{1}$, and $\phi_{0}$ can be chosen by varying $l_{1}$.

Reflectivities can also be reduced by taking a single $u l$ trathin film on a low-density backing. For example, with $10-\AA \mathrm{Fe}$ on $\mathrm{Be}$ the reflectivity is only $\approx 1 \%$ in a region where a pure $\mathrm{Fe}$ mirror has a $30 \%$ reflectivity. A true GIAR film is possible by using two layered UT films separated by a low-density $\lambda / 4$ film. Because all contributing reflection amplitudes are small, a very deep interference minimum should be possible even without exact impedance matching.

The greatest versatility is offered by multilayer techniques. By going to multilayer coatings, the choice of possible materials for GIAR films is greatly extended, and by "thickness tuning," it is possible to arbitrarily specify the operating angle $\phi_{0}$. Furthermore, with an appropriate choice of materials, it is possible to greatly broaden the angular and frequency regions of strong suppression.

A limiting case of multilayer films is a tapered GIAR film. Here the combined effects of a slow gradient increase of electron density and destructive interference give a strongly reduced reflectivity-essentially just that of the upper low-density interface. Such films may be of limited practical use, but they are useful for understanding the effects of surface and volume roughness, which we examine in a following paper. ${ }^{5}$

\section{ACKNOWLEDGMENTS}

This work was partially supported by the National Science Foundation, Grant No. DMR-80-15706, and the Deutsche Forschungsgemeinschaft and the Bundesministerium für Forschung und Technologie, Grant No. 05269GU.
1J. P. Hannon et al., preceding paper, Phys. Rev. B 32, 5068 (1985), henceforth referred to as paper I.

${ }^{2}$ H. A. Macleod, Thin Film Optical Filters (Elsevier, New York, 1969), and references therein.

${ }^{3}$ A. Vasicek, Optics of Thin Films (North-Holland, Amsterdam,
1960).

${ }^{4} \mathrm{M}$. Bron and E. Wolf, Principles of Optics (Pergamon, New York, 1959), pp. 50-69.

${ }^{5}$ J. P. Hannon, J. T. Hutton, G. T. Trammell, E. Gerdau, and R. Rüffer, paper VI (unpublished). 